\title{
Gradhiva
}

GRADHI

Revue d'anthropologie et d'histoire des arts

$8 \mid 2008$

Mémoire de l'esclavage au Bénin

\section{Tours et détours des mémoires familiales à Ouidah}

La place de l'esclavage en question

Émile-Désiré Ologoudou

\section{OpenEdition}

Journals

Édition électronique

URL : http://journals.openedition.org/gradhiva/1179

DOI : 10.4000/gradhiva. 1179

ISSN : 1760-849X

Éditeur

Musée du quai Branly Jacques Chirac

Édition imprimée

Date de publication : 15 novembre 2008

Pagination : 80-86

ISBN : 978-2-915133-94-3

ISSN : 0764-8928

Référence électronique

Émile-Désiré Ologoudou, «Tours et détours des mémoires familiales à Ouidah », Gradhiva [En ligne],

8 | 2008, mis en ligne le 15 novembre 2011, consulté le 19 avril 2019. URL : http://

journals.openedition.org/gradhiva/1179; DOI : 10.4000/gradhiva.1179

(c) musée du quai Branly 


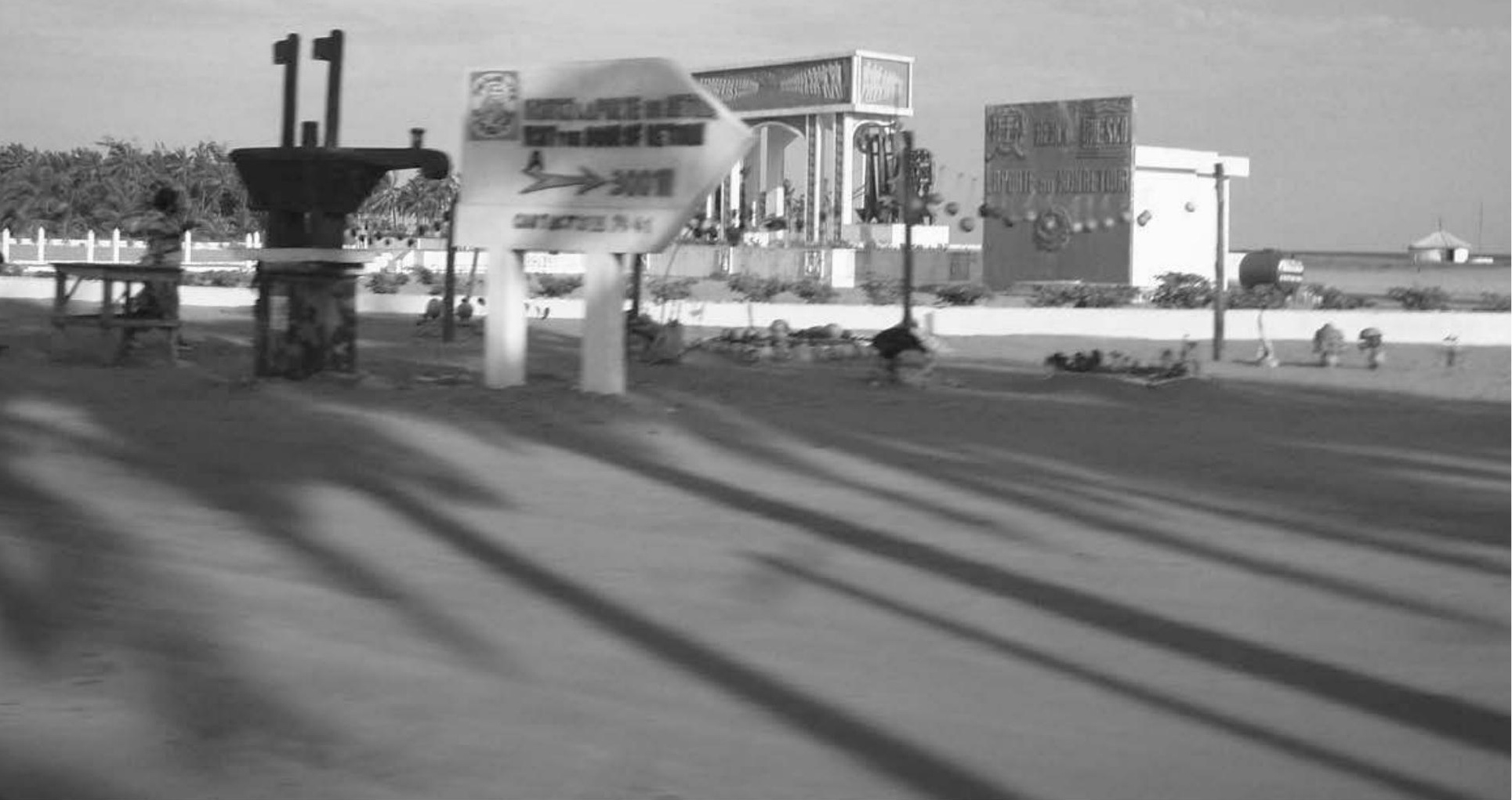

Site de la Porte du Non-Retour, plage de Ouidah, 2005.

Euvres de Fortuné Bandeira, Dominique Gnonnou « Kouas » et Yves Kpède. Photo G. Ciarcia. 


\title{
DOCUMENTS ET MATÉRIAUX \|Émile-Désiré Ologoudou
}

\section{Tours et détours des mémoires familiales à Ouidah}

\author{
La place de l'esclavage en question
}

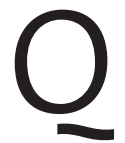

uand on a élu domicile à Ouidah, on reçoit beaucoup d'annonces imprimées de messes de huitaine ou autre périodicité, qui sont célébrées dans les familles en mémoire de tel ou tel défunt. Les presses artisanales installées de vieille date au cœur de la ville, et aujourd'hui disparues pour la plupart, n'ont jamais eu d'autre tâche que ces annonces nécrologiques et autres menus travaux. En réfléchissant à la transmission orale de l'expérience de l'esclavage vécue par nos ancêtres, je me suis interrogé sur la relation ambiguë entre ces imprimés funéraires et une mémoire des temps révolus.

Seules pouvaient autrefois commander des faire-part bordés de noir sur blanc des familles non seulement aisées, mais qui se faisaient une certaine idée de leur importance et de la grandeur de leur honneur mis à l'épreuve par l'exigence d'enterrer dignement les leurs. Peu à peu, j'ai fait de ces imprimés déclinant les noms et les titres de l'ancêtre éponyme et de ses alliés une source lointaine de mes recherches sur les grandes collectivités lignagères de Ouidah. Car, manifestement, lorsqu'il s'agit d'évoquer le passé de l'esclavage et de la traite atlantique, la mémoire de ces familles dont ces faire-part sont des supports publics peut aussi devenir un véritable labyrinthe pour leurs descendants et, à plus forte raison, pour des chercheurs toujours trop curieux: le flou autour des ascendances réelles est parfois soigneusement entretenu lorsque tel ou tel épisode de l'histoire familiale est jugé désobligeant.
Souvent, ainsi, à la parole des descendants des maîtres et seigneurs fait écho le silence et la gêne des descendants des dominés de jadis, qui peuvent se contenter de faire de vagues allusions à la déchéance " momentanée " que leurs ancêtres ont endurée lors de leur passé de captifs. La politique des grandes collectivités fon (ou assimilés) propriétaires d'esclaves envers ces derniers peut en effet être considérée comme ayant été assimilationniste, et nombre de lignées d'origine servile se sont ou ont été partiellement assimilées aux lignages de leurs maîtres à l'époque de la domination fon sur Ouidah, entre la seconde partie du xviII ${ }^{\mathrm{e}}$ siècle et la fin du xIx siècle. Or, avec la fin de l'esclavage local et le début de la domination française, dans la dernière décennie $\mathrm{du}$ xIx ${ }^{\mathrm{e}}$ siècle, la dynamique assimilationniste du système esclavagiste fon s'est trouvée en panne, et les revendications du droit à organiser des cérémonies de naissance et de mort selon les rites de la région d'origine sont montées en puissance dans les lignées issues d'esclaves qui pouvaient être en voie d'intégration aux lignages de leurs maîtres ou seigneurs. À cette époque, toutefois, certaines familles passées par l'expérience de l'esclavage avaient déjà conquis une certaine indépendance ou s'étaient vu affranchies, et avaient pour certaines elles-mêmes acquis des esclaves, signe de leur réussite sociale et de leur aisance matérielle.

Dans les pages qui suivent, je voudrais évoquer les mémoires de l'esclavage et leur gestion dans deux collectivités familiales de Ouidah : la collectivité Tchibozo, 
tout d'abord, " grande famille " autrefois propriétaire de nombreux esclaves comme les collectivités Gnahoui et Quenum ${ }^{1}$; la famille Ologoudou, ensuite, à l'origine de condition servile, mais qui connut un affranchissement et une ascension sociale rapides. À travers les évocations contrastées de ces cas, je montrerai la pluralité et les ambiguités des mémoires de l'esclavage dans le Ouidah d'aujourd'hui.

\section{Les mémoires inégales de la collectivité Tchibozo}

Selon les narrations autorisées dans la famille Tchibozo, l'ancêtre éponyme aurait été envoyé par le roi du Dahomey, de son village natal Linsinlin (à proximité d'Abomey), pour surveiller et conquérir des terres à Ouidah. Après avoir effectué des razzias dans plusieurs autres régions du Dahomey, Tchibozo arrive à Ouidah sur la recommandation, dit-on, du prince Tomètin, proche du roi Guézo. Avec le temps, Tchibozo devient un oluwa (maître et seigneur, en yoruba), comme Affimitin Gnahoui et Azanmado-Houenou (dit Quenum), c'est-àdire un cabécère anobli, plus ou moins agrégé aux princes de sang, et propriétaire de terres et d'esclaves attachés à son service.

Comme j'ai pu le remarquer au cours de mes enquêtes menées dans les dernières années, de nos jours, l'écriture de l'histoire du lignage ne laisse personne indifférent dans les seize « maisons » ou lignées qui forment aujourd'hui la collectivité Tchibozo, au sein de laquelle il faut en fait distinguer entre les lignées qui se veulent directement reliées à l'ancêtre éponyme par le sang, un deuxième groupe qui s'affirme de préférence comme étant issu des descendants des premiers compagnons de migration vers la côte, et enfin un troisième groupe de lignées, issues d'esclaves d'origine yoruba liés à l'ancêtre par le travail dans les champs et les fermes. Et, selon les origines, on peut observer dans la collectivité Tchibozo comme dans d'autres collectivités du même genre à Ouidah diverses stratégies qui, notamment à travers les mariages conclus entre lignées au sein de la même collectivité, visent le maintien de la primauté, ou au contraire le rapprochement du noyau «noble » du lignage.

Rien d'étonnant à ce que, dans ce contexte où plusieurs niveaux de parenté se croisent et s'affrontent, les points de vue abondent et divergent dès qu'on doit narrer l'histoire des Tchibozo et préciser les supposées «identités réelles » de ses membres. On se rend vite compte alors que, ici comme dans d'autres familles, l'histoire est un enjeu, qu'elle soit rapportée par les détenteurs de titulatures et de "sièges " dûment reconnus du " centre» de la collectivité ou par les descendants des premiers compagnons de migration de l' « homme fort " devenu ancêtre apical ou, à plus forte raison, par les individus issus des esclaves qui parfois ont connu des ascensions fulgurantes, à faire pâlir leurs maîtres d'hier. Selon les positions d'énonciation, les interlocuteurs soulignent ainsi tantôt l'importance des faits d'armes de l'ancêtre fondateur et le caractère impressionnant des possessions dont on lui attribue l'acquisition, ou plutôt le rôle fondamental joué par tel ou tel compagnon des débuts, responsable des cérémonies funéraires dans l'ensemble de la collectivité ou devin attitré de celle-ci, par exemple.

Mais on notera aussi que ces diverses mémoires lignagères ne sont pas seulement, ici comme dans bien d'autres familles de Ouidah d'ailleurs, des mémoires d'hommes. Dans la lignée Houndedji, par exemple, on revendique une place éminente dans l'histoire de la collectivité en raison de la place centrale occupée, au cœur de celle-ci, au xIX ${ }^{e}$ siècle, par une femme de cette lignée qui, à travers une série de mariages, a mis au monde des enfants pour diverses autres lignées de la collectivité et détenu tous les secrets généalogiques, de même qu'elle a lutté pour réunir ou conserver dans les mains des Tchibozo de nombreux grands domaines. Ainsi, le nom Houndedji Tchibozo est aujourd'hui fièrement arboré, parce que cette femme réputée « de tête » a eu de nombreux descendants qui se sont distingués par leur audace et leur âpreté au gain. En outre, les descendants de cette femme sont aussi fiers de leur ascendance parce qu'ils sont, comme on dit à Ouidah dans de tels cas, chokoto kpo, kan kpo, c'est-à-dire «à la fois pantalon et ceinture ", ce qui est une façon conventionnelle de réclamer sa filiation dans un lignage à la fois en ligne paternelle et maternelle, et ainsi le cumul d'une double légitimité dans la collectivité familiale.

Enfin, bien souvent, les descendants de lignées d'origine servile ne se font aucune illusion sur les différences de classe masquées par le partage du nom Tchibozo avec les autres lignées. En fait, sous cette identité générique percent vite les « vrais " noms antérieurs à l'es-

1. Les généalogies de ces familles ont déjà fait l'objet de travaux comme celui de Joseph-Marie Covi sur les Affimitin Gnahoui (Les Grandes Collectivités familiales de Ouidah dans leurs rapports avec les Akwèxonu : le cas de la famille Gnahoui-Affimitin avec ses Yoruba-Nago de 1850 à l'an 2000, Université nationale du Bénin, mémoire de maîtrise en histoire et archéologie) et celui, en cours, de Salomon Dossou sur les rapports entre les Azanmado Houenou (alias Quenum) et leurs esclaves. 
clavage, dont la mémoire a souvent été conservée. En outre, chaque groupe ayant fait souche est aujourd'hui périodiquement amené à sacrifier à des cultes et des rites différents selon les origines antérieures à l'esclavage revendiquées et les formes de culte aux ancêtres qu'elles impliquent. Ainsi, si rien ne peut effacer aujourd'hui chez les Tchibozo la mémoire de l'esclavage, celui-ci est aussi régulièrement euphémisé par les lignées issues d'esclaves ou de dépendants. Le rappel d'une condition servile passée peut d'ailleurs être récusé d'une manière si vive et dans une si large mesure que le chercheur se voit obligé de ne pas trop solliciter l'évocation des faits du passé et de se contenter de certaines menues indications qu'on lui transmet.

Est-ce à dire que le souvenir de l'esclavage local est globalement tu et occulté dans la société ouidanaise d'aujourd'hui? Pas exactement. Très récemment, une agitation fébrile a ainsi accompagné l'initiative d'un prêtre et d'une religieuse catholiques appartenant à la famille Tchibozo. Je n'ai pas pu les rencontrer en personne, mais un certain nombre de mes informateurs se sont faits l'écho de leur passage assez bruyant, dans la mesure où les deux religieux cherchaient à faire valider une version de l'histoire de la collectivité considérée comme moderniste par les dignitaires et diverses autres personnalités lignagères, vieilles et jeunes. En particulier, les notables formés à l'école de l'observance traditionnelle de la hiérarchie familiale se rendaient compte de la menace qui pesait, dans la version proposée par les deux religieux, sur la primauté conférée traditionnellement à l'origine des différentes lignées. Le parti des notables s'opposait donc à toute tentative de «brouillage » des différences de statut entre les individus issus des branches d'origine servile et les descendants des maîtres d'antan. On le voit : si, aujourd'hui, tout le monde peut signer indistinctement avec le nom Tchibozo, la mémoire des inégalités passées - en particulier de celles héritées de l'esclavage - est, dans certains contextes, soigneusement entretenue et susceptible d'être rappelée. Il y a toujours, de ce point de vue, Tchibozo et Tchibozo.

\section{Mémoire de l'esclavage et sentiment de distinction dans la famille Ologoudou}

De nos jours, de façon contrastée par rapport à cette gestion discrète de la diversité culturelle et des hiérarchies sociales héritées du passé de l'esclavage, les descendants de la famille Ologoudou versés, comme moi-même, dans l'histoire lignagère semblent recon- naître qu'aucun Yoruba (ou Nago) n'est venu en promenade de santé à Ouidah. Comme les autres Yoruba arrivés dans la ville, les Ologoudou savent qu'ils ont été des captifs, c'est-à-dire des gens qui ont été surpris dans leurs villes, villages ou hameaux du monde yoruba et contraints de suivre les négriers ou leurs intermédiaires vers une destination inconnue.

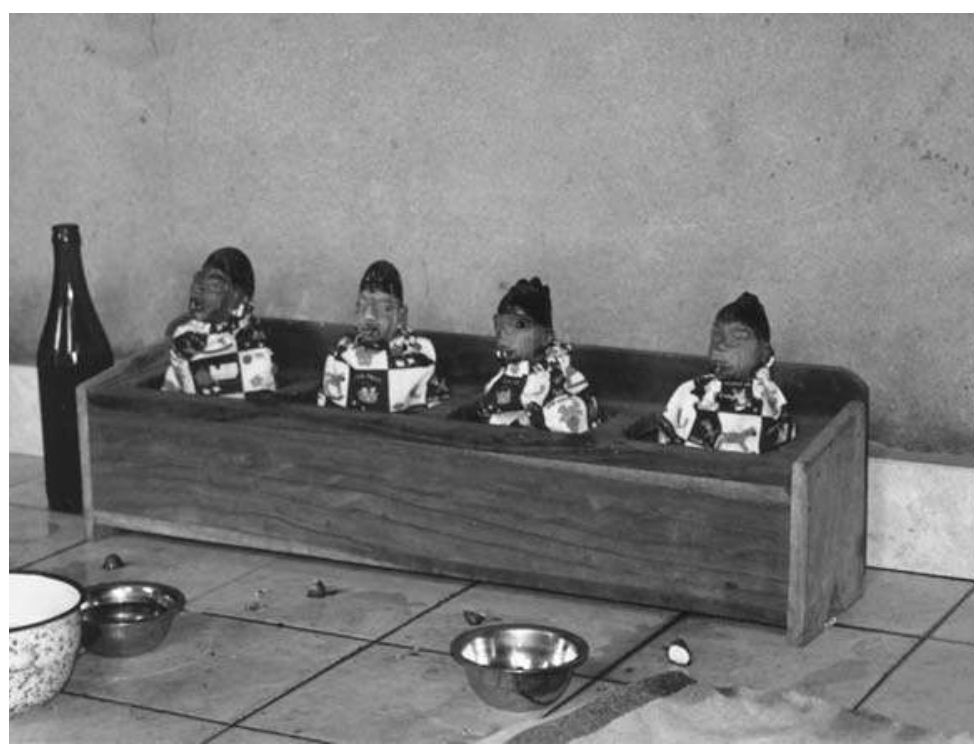

Fig. 2 Véritables sacra, et supports de la mémoire familiale des origines, les vieilles statuettes du culte des jumeaux dans la concession Ologoudou, apportées depuis le pays ègba par l'ancêtre fondateur du lignage, dans la première moitié du XIX siècle, Ouidah, 2003. Photo J. Noret.

L'esclavage local, toutefois, n'impliquant pas la déportation outre-Atlantique, ne s'est pas révélé incompatible avec le souvenir et la persistance d'une mémoire des origines antérieures à l'expérience du déplacement forcé et de la servilité. On sait ainsi aujourd'hui à Ouidah que des princes comme des roturiers ont été amenés comme captifs dans la ville, et il n'y a pas de contradiction entre le fait d'avoir été captif et celui de se dire, par la suite, noble à part entière, soucieux en tout cas de récupérer le plus rapidement possible cette identité « originelle ». L'expérience de l'esclavage est loin d'impliquer nécessairement une amnésie quant aux origines familiales. Certaines populations de Ouidah ont ainsi pu s'affranchir à bien des égards de la tare représentée par la capture et la réduction en esclavage pour gagner une position sociale respectable envers leurs anciens maîtres. D'ailleurs, d'anciens esclaves affranchis sont parfois devenus eux-mêmes des négriers, ou tout au moins des propriétaires d'esclaves.

L'ancêtre éponyme des Ologoudou, Chohountan Ologoudou, détaché des siens à Abeokuta, au cœur du 
pays ègba (un «sous-groupe " yoruba), à une petite centaine de kilomètres à l'est de Kétou, s'installe à Ouidah, autour de 1830, dans la dépendance immédiate de la dame Chica daho ${ }^{2}$ (grande), une des filles aînées du négrier brésilien Francisco Felix de Souza, connu aussi sous le titre de Chacha $\mathrm{I}^{\mathrm{er}}$. Chohountan arrive à Ouidah sans avoir, semble-t-il, connu une capture fort violente puisqu'il amène avec lui, selon ce qui me fut transmis, son Ifa (divinité de la divination) personnel (lequel ne sera pas, contrairement à l'usage le plus courant, détruit à sa mort, et est devenu une sorte de patrimoine lignager inaliénable), ainsi que la divinité Gbadu et un morceau de pierre éclatée de la montagne dite Olumon que chaque exilé ègba cherche à prélever sur les collines d'Abeokuta pour en faire une de ses premières divinités dans le pays d'accueil. À Ouidah, Chohountan, peuton supposer, découvre un univers mercantile où tous les arrivants sont inspectés du point de vue de leur santé et de leur force physique, mais aussi de leurs compétences et de leurs savoirs. Classés sur une liste d'aptitude-qui répartit les futurs fermiers et les artisans en ville, ceux qui pratiqueront de petits métiers comme les maçons, les menuisiers, les ébénistes, les ferronniers, les blanchisseurs et les coiffeurs -, les devins (bokonon en fon, babalawo en yoruba) comme Chohountan sont prisés pour leurs connaissances divinatoires et thérapeutiques.

Chohountan s'intègre ainsi aux cercles influents des bokonon de Chacha I ${ }^{\mathrm{er}}$, dont certains ont habité d'abord le quartier d'Agbodji comme lui, avant d'être recasés à Zomaï suite au nombre toujours croissant d'esclaves Yoruba et Mahi et aux nombreux incendies dus à la promiscuité. Chohountan s'est donc vite écarté d'Agbodji, dès qu'il a pu disposer dans le quartier Zomaï d'une concession assez vaste pour accueillir d'autres compagnons, aujourd'hui réunis dans le même culte du souvenir autour de l'autel central de la maison Ologoudou - où l'on pratique un culte des ancêtres connu de type oro, les Ologoudou étant «adeptes d'oro ${ }^{3}$.

Ici, au cours de célébrations annuelles, on rappelle chacun des noms des "pères fondateurs " (les hommes comme les femmes) par ordre de décès devant un autel central demeuré unique jusqu'à la troisième génération, quand baba Awakan - André Ologoudou, mon père - dans la seconde partie des années 1950, décentralisa les autels ancestraux de telle sorte que les lignées issues des compagnons que Chohountan avait attirés à lui puissent invoquer à leur aise tous les leurs sans restriction. En effet, Chohountan, en raison des compétences divinatoires qui lui furent reconnues, a attiré à lui un certain nombre de compagnons auxquels il donna des emplacements au sein même de la concession qui lui avait été attribuée (par la collectivité de Souza). Sans être des esclaves (mèhiho, littéralement " personne achetée ") ni des dépendants, ceux-ci étaient à proprement parler des compagnons. Auprès de Chohountan vinrent ainsi vivre Akilabi, un Ėgba-Owu de tradition elegun ("adepte d'egun ", une forme de culte aux ancêtres impliquant la sortie de masques bien connus chez les Yoruba), puis Atchade, un homme originaire de Kétou et de tradition oloro (" adepte d'oro »), et ensuite Olowokou, d'appartenance elegun Èsho-Ikoyi et de même mère que les descendants de Chohountan Ologoudou, lequel avait épousé la mère d'Olowokou en secondes noces. Chaque année, lors des cérémonies organisées en souvenir des ancêtres, ces noms sont évoqués à l'autel central de la famille Ologoudou, avant que les lignées issues des compagnons de Chohountan n'aillent évoquer leurs défunts séparément.

Chohountan Ologoudou exerça donc une certaine influence sociale, et il me semble important de souligner que les Ologoudou n'ont pas eu besoin d'affranchissement officiel avant de se sentir libres et de se comporter en hommes libres; ceux de la première génération, avec Chohountan (arrivé vers 1830), et de la deuxième, avec Abatan (né vers 1850, mort en 1934), s'inscrivaient dans la catégorie sociale très influente des devins (babalawo et bokonon) au service de Chacha $\mathrm{I}^{\mathrm{er}}$ (ce surnom donné à Francisco Felix de Souza devint le titre des chefs du lignage de Souza) et de sa famille.

Famille de type maraboutique ayant entretenu, au cours des deux premières générations, un temple-école qui connut différentes promotions d'internes venus là pour apprendre la divination par Ifa, la famille Ologoudou a ensuite évolué en direction du secteur privé et de la fonction publique ${ }^{4}$, avant que les blocages postcoloniaux n'entraînent, de gré ou de force, la diversifi-

2. Le prénom Chica est en fait un diminutif de Francisca, tandis que l'adjectif fon daho fait allusion au rang de fille aînée. Tandis que d'autres ont, suite à leur dépendance envers la Chica daho, commencé signer du nom de Sikè, transformation de Chica, les Ologoudou ont pu garder leur nom yoruba à travers le temps.

3. Dans ce culte aux ancêtres, contrôlé par une société initiatique masculine, ces derniers se manifestent le plus souvent sous la forme de voix graves ou aiguës inarticulées, émises depuis les espaces réservés aux initiés et audibles à quelques dizaines de mètres aux alentours - parfois davantage lorsque, en contexte villageois le plus souvent, le culte est organisé autour d'un bois sacré d'où les voix des oro peuvent se faire entendre à quelques centaines de mètres.

4. «L'école est la palmeraie [une forme de capital par excellence dans la région] que je laisse à chacun de vous », avait l'habitude de dire mon père à ses enfants. 


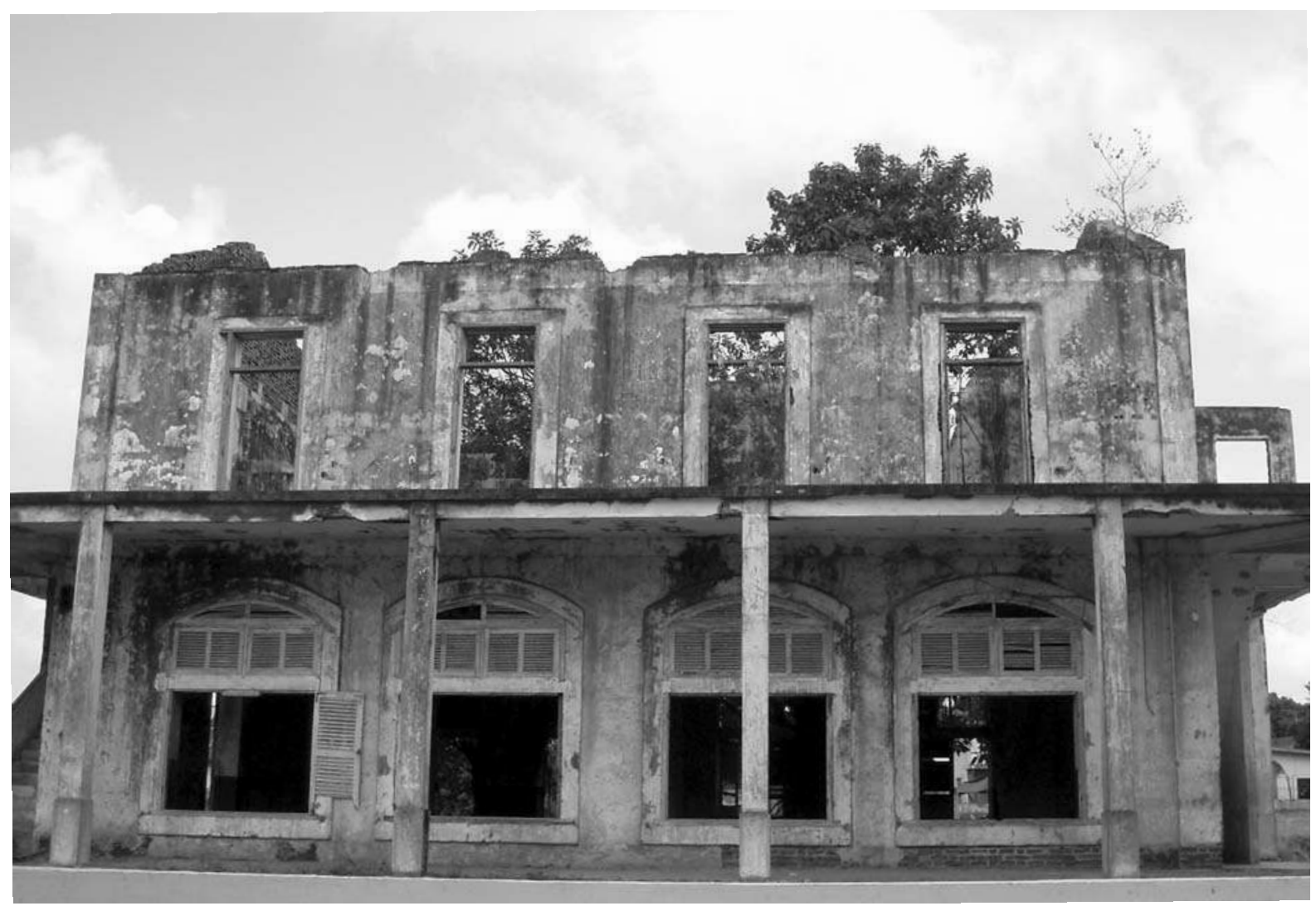

Fig. 3 Ruines d'une maison au style architectural dit « afro-brésilien », Ouidah, 2005. Photo G. Ciarcia.

cation des débouchés dans les générations suivantes. Sans avoir été à l'origine des propriétaires terriens ou de gros possédants, les Ologoudou, qui connaissent aujourd'hui leur quatrième baba en la personne de mon frère aîné, $b a b a$ Ola Victor, n'en sont pas moins confrontés à divers conflits familiaux liés, d'une part, à des ruptures avec les "coutumes" (tous les Ologoudou ne s'investissent plus dans le culte ancestral, un certain nombre d'entre eux refusant en particulier de le faire en raison de leur identité chrétienne) et, d'autre part, à une forme de fracture sociale au sein de la famille, dont certains membres ont intégré les plus hautes sphères de l'administration et, au fond, la bourgeoisie, tandis que d'autres sont restés analphabètes et à l'écart des circuits modernes de formation et de promotion sociale.

Mais c'est sur deux traces manifestes laissées dans la mémoire familiale par l'insertion passée du lignage dans une société esclavagiste que je voudrais conclure cette évocation. La première renvoie à la dépendance initiale de la famille à l'égard de la collectivité de Souza. En effet, signe de la persistance des classements sociaux hérités de l'esclavage, lorsque mon père, André Ologoudou, petit-fils de Chohountan, s'apprêtait à entrer à l'école primaire supérieure Victor-Ballot de Porto-Novo, sorte d'antichambre de l'administration coloniale, quelques amis bien intentionnés lui suggérèrent d'abandonner son nom, de revendiquer explicitement la proximité historique de sa famille avec celle des de Souza (dont on connaît l'importance, Francisco de Souza ayant été historiquement le plus important négrier installé à Ouidah et le fondateur dans la ville de la communauté "brésilienne »), et donc de signer désormais du nom de « de Souza » et non plus de celui d'Ologoudou : le patronyme de Souza était considéré comme susceptible d'ouvrir encore bien des portes en 1925. Mon père toutefois refusa net et poussa le zèle jusqu'à exiger de son père Abatan qu'il lui fasse les scarifications mèrin-mèrin portées d'ordinaire par les Ègba (le groupe "ethnique " d'origine de Chohountan). Les scarifications avaient cependant déjà été abandonnées à la génération précédente, née à Ouidah, et Abatan répondit à son fils : "Comment le pourrais-je si tard, n'ayant pas été moimême scarifié au visage?"

La seconde trace renvoie à la fois à l'importance de la parenté par les femmes dans la société de Ouidah et au caractère profondément structurant des compagnon- 


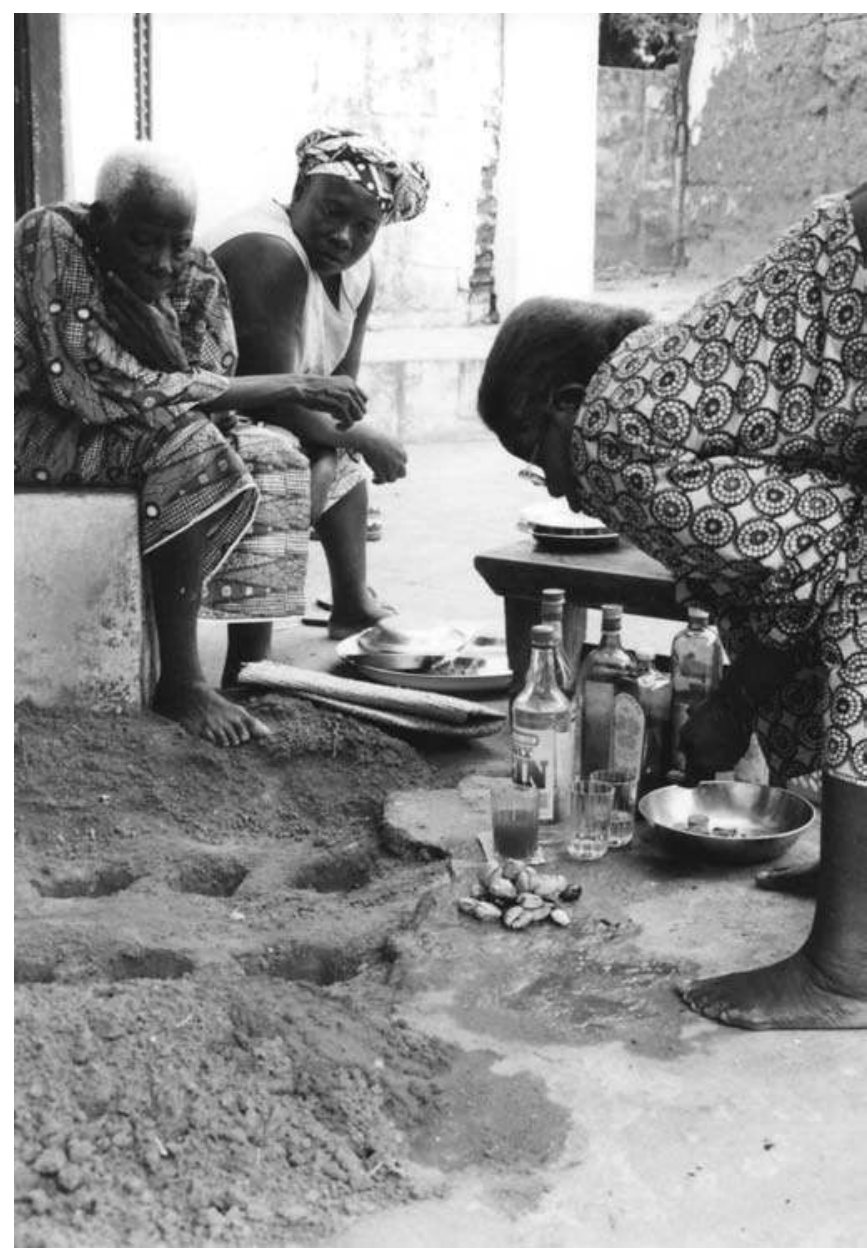

Fig.4 Rites lignagers de naissance dans une famille proche des Ologoudou, Ouidah, 2003. Photo J. Noret.

nages de la société du XIX ${ }^{\mathrm{e}}$ siècle. Mon père, qui occupa le trône de chef de lignage sous le nom de baba Awakan, était le seul fils de son père, baba Abatan, à avoir des enfants. Ayant gardé de cette expérience une conscience aiguë de la fragilité potentielle de la continuité familiale, il nous disait toujours, à mes frères et à moi, qu'en cas de manque de descendants mâles et donc de vide du trône lignager, ce serait du côté du fils en premières noces de la femme de Chohountan, qu'il fallait chercher pour trouver un baba (chef) au lignage. En effet, je l'ai dit, la femme de Chohountan, Agounke ou Agnikè, que selon la tradition familiale il avait achetée à prix d'or (elle était esclave) pour l'épouser en secondes noces, avait un fils d'un premier lit, Olowokou. En se mariant et en donnant des enfants dans la maison Ologoudou, Agnikè s'affranchit de son statut, mais son fils Olowokou resta esclave. De même mère que les descendants de Chohountan et progressivement intégré au lignage, il finit toutefois par être considéré comme l'un de ses membres puisque mon père nous suggéra, au milieu $\mathrm{du} \mathrm{xx}^{\mathrm{e}}$ siècle, de nous tourner vers ses descendants en cas de pénurie de descendants masculins parmi les descendants directs de Chohountan.

Le Bénin est aujourd'hui une démocratie postcoloniale et un État de droit. Pourtant, les réticences et les difficultés fréquemment rencontrées lorsque l'on évoque la place de l'esclavage montrent clairement que le contexte démocratique actuel n'est pas suffisant pour lever les interdits mémoriels qui entourent cette question complexe, et qui freinent aujourd'hui encore la possibilité d'une histoire complète et libérée. En effet, dans les grandes comme dans les petites familles de Ouidah, dissensions et divisions internes, voire conflits ouverts, rendent la parole difficile et génèrent bon nombre de réticences. En sus, le souci de la grandeur lignagère veut souvent que, dès que le nombre des descendants dépasse la centaine, nos gens se disent déjà en royauté. Étant des « rois " pour eux-mêmes et les leurs, les notables et autres dignitaires rechignent souvent à livrer leurs commentaires au chercheur, dont la curiosité peut agacer.

Enfin, dans le contexte patrimonial actuel et à un niveau plus général, tout se complique avec un dilemme qu'on pourrait exprimer en ces termes : entre mémoire volontairement amnésiée, excès de mémoire, oubli, ressentiment, commercialisation de la mémoire, politisation de la mémoire, comment vivre aujourd'hui cette histoire commune et divisée ? Une telle question est évidemment fort complexe. Mais pour le sociologue, en tout cas, mieux comprendre cette immense tragédie et ses conséquences demande, à tout le moins, d'interroger la "tradition » orale moins comme une répétition invariante que comme un espace où la production des généalogies participe de transformations mémorielles.

Chargé de cours émérite de l'Université nationale du Bénin, aujourd'hui université d'Abomey-Calavi (UAC) emileologoudou@yahoo.fr 